\title{
Effects of perception and production trainings on the production of English vowels by French native learners
}

\author{
Jennifer Krzonowski ${ }^{1}$, Emmanuel Ferragne ${ }^{2}$, François Pellegrino ${ }^{1}$ \\ ${ }^{1}$ Laboratoire Dynamique du Langage UMR5596 CNRS/Université de Lyon, France \\ ${ }^{2}$ CLILLAC-ARP EA3967, Université Paris Diderot, France \\ https://doi.org/10.36505/ExLing-2015/06/0010/000247
}

\begin{abstract}
This study examined the effect of two different trainings on the production of English vowels $(/ \mathrm{i}: /, / \mathrm{I} /, / \mathfrak{x} /, / \Lambda /$ and $/ \mathrm{a}: /)$ by French learners. Forty-eight French first-year students, who had learned English in school only, were divided into three groups receiving either 5 sessions of perceptual training (PE-Group), or 5 sessions of production training (PR-Group) or no training (C-Group). They were recorded at pretest and post-test with a reading task of $/ \mathrm{bVd} /$ words, and their performance was evaluated by discriminant analysis based on sex-specific models trained on native speakers productions. The results show improved classification rates for the vowels $/ \Lambda$ / and /I/ in the PR-Group and only for /i:/ in the PE-Group. No improvement was observed for the C-Group.
\end{abstract}

Key words: L2 phonology acquisition, vowel production, training

\section{Introduction}

Phonetic trainings have already shown their efficiency for improving perception and production performance for second-language learners both for consonant (Bradlow, 2008, Iverson, Hazan and Bannister, 2005, Pruitt, Jenkins and Strange, 2006) and vowel categories (Aliaga-Garcia, 2010, Iverson and Evans, 2009, Iverson, Pinet and Evans, 2011, Lambacher, Martens, Kakehi, Marasinghe and Molholt, 2005). Among the studies carried out on the acquisition of second language vowels, very few relate to the acquisition of English vowels by French learners and performance assessment is often restricted to perception. Iverson, Pinet and Evans (2011) tested the effect of a classical High Variability Perceptual Training on the acquisition of English vowels by French speakers. Their results in production showed significant improvement only for $/ \mathrm{i}: /, / \varepsilon \mathrm{I} /$ and $/ \alpha \mathrm{Y} /$.

Our study investigates the effect of two different types of training (perception and production) on the production of English vowels by French learners. We focused on two regions of the vowel space that are known to cause difficulties for these learners: /i: - I/ and / $\mathfrak{x}-\Lambda-\mathrm{a}: /$. These vowels are located in the vowel space where there is only one category in French but two (/i: - I/) or three $(/ \mathfrak{x}-\Lambda-\mathrm{a}: /)$ in English.

ExLing 2015: Proceedings of 6th Tutorial and Research Workshop on Experimental Linguistics, 26-27 June 2015, Athens, Greece 


\section{Procedure}

The study followed a pre-test / training (five 1-hour sessions on consecutive days) / post-test paradigm. The test sessions included a production task involving three $/ \mathrm{bVd} /$ words for each vowel category.

Forty-eight first-year students of English were divided into 3 groups: one group received a high variability perceptual training (PE-Group)(Iverson, Pinet and Evans, 2011), a second one received a production training (PR-Group). A last control group (C-Group) received no training but listened to audiobooks in English between test sessions.

The training material consisted of recordings by native speakers of British English. Ten CVC minimal pairs for each of the three contrasts involved (/i:/$/ \mathrm{I} /, / \mathfrak{x} /-/ \Lambda /$ and $/ \Lambda /-/ \mathrm{a}: /)$ were produced by twelve native speakers (6 women) from the South East of England.

The first three training sessions involved one of the three contrasts each, and the last two sessions combined all contrasts. For the PE-Group, the first three sessions contained 2AFC identification and AX discrimination tasks with feedback, while the last two sessions were composed of 5AFC identification and oddity discrimination tasks with feedback.

The PR-Group performed a word repetition task with feedback in all sessions. The feedback was visually given from a real-time acoustic analysis of the participants' production (vowel formants and duration) compared to those of the native speakers of the same sex. The result of an automatic classification of the vowel based on a sex-specific model trained on native English productions was also displayed.

\section{Data analysis}

Our expectations were that, if the training was effective, automatic vowel classification would improve between the pre-test and the post-test. The first two formant values measured at vowel midpoint were computed, Barktransformed, and z-scored (independently for F1 and F2) for each speaker (Ferragne and Pellegrino, 2010). The natives' formant values were used to train a linear discriminant classifier with one model for women and one for men. Then the learners' vowels were automatically classified based on the appropriate model.

\section{Results}

The overall correct classification score for the pre-test is $78 \%$. Important discrepancies between vowel categories are found: $/ \mathrm{i}: /: 71 \%, / \mathrm{I} /: 77 \%, / \mathfrak{x} /$ : $92 \%, / \Lambda /: 55 \%$, /a: $/: 94 \%$. Clearly, most misclassifications come from the vowel $/ \Lambda /$. Classification rates for all vowel categories taken together improved between pre-test and post-test for the PR-Group (7 percentage points - pp) and 
the PE-Group (7 pp), both reaching statistical significance according to a binomial test (respectively, $p=0.02$ and $p<0.01$ ), whereas it dropped for the CGroup (-6 pp, $p=0.03)$.

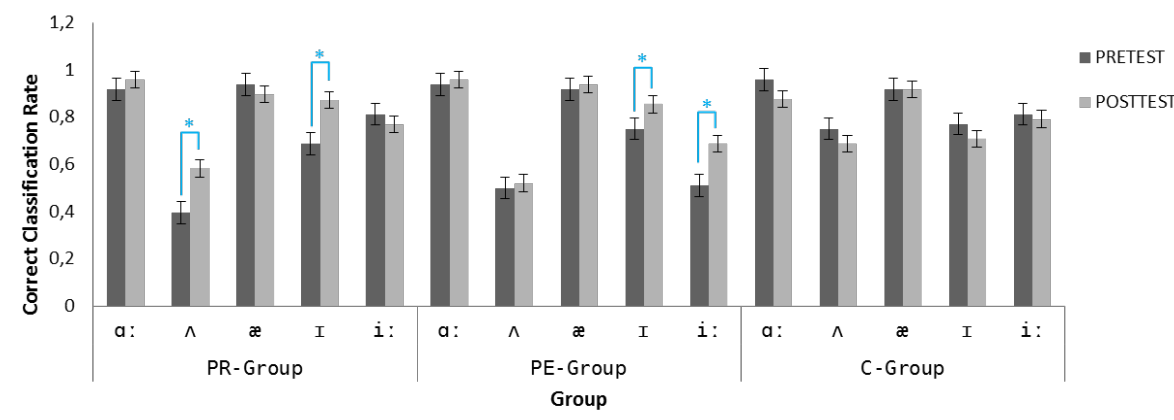

Figure 1. Classification rates for all vowels at pre-test (dark bars) and post-test (light bars) for each group with error-bars.

Now focusing on the vowels (see Figure 1), classification rates for $/ \Lambda /$ increased by $19 \mathrm{pp}$ for the PR-Group, which was found to be a significant improvement $(p=0.007$ ). For the PE-Group, the increase was only 2 pp (not significant). The C-Group decreased by $6 \mathrm{pp}$ (not significant). The vowel /I/ was also significantly better categorized at post-test for the PR-Group, increasing by $18 \mathrm{pp}(p=0.006)$. The PE-Group's performance also increased for this vowel (10 pp, $p=0.012)$. As for $/ \Lambda /$, at pre-test, the vowel $/ \mathrm{I} /$ was often misidentified as /i:/, and less often so at post-test. The PE-Group showed another significant improvement for the vowel $/ \mathrm{i}$ / (18 pp, $p=0.014)$.

\section{Conclusion}

There is therefore evidence that the production training was effective for the acquisition of $/ \Lambda /$ but not the perception training. Both training groups improved on the production of $/ \mathrm{I} /$, but only the PE-Group performed better for the entire /i: - I/ contrast. These results will be compared to perceptual scores in a follow-up study. The different patterns observed between the training groups could be correlated with the perception patterns for these two contrasts: it may be that the /i:/-/I/ contrast is more difficult to perceive than $/ \mathfrak{x} /-/ \Lambda /$ and that a perceptual training is more appropriate for the former contrast to help French speakers. 


\section{Acknowledgements}

This research was supported by an IUF grant to Emmanuel Ferragne and by the ASLAN Labex.

\section{References}

Aliaga-Garcia, C. 2010, Measuring perceptual cue weighting after training: A comparison of auditory vs. articulatory training methods, K. Dziubalska-Kołaczyk, M. Wrembel and M. Kul (eds.), New Sounds 2010, Poznan, Poland

Ferragne, E. and Pellegrino, F. 2010, Formant frequencies of vowels in 13 accents of the British Isles, Journal of the International Phonetic Association, 40, 1-34

Iverson, P. and Evans, B. G. 2009, Learning English vowels with different firstlanguage vowel systems II: Auditory training for native Spanish and German speakers, Journal of the Acoustical Society of America, 126, 866-877

Iverson, P., Hazan, V. and Bannister, K. 2005, Phonetic training with acoustic cue manipulations: A comparison of methods for teaching English /r/-/1/ to Japanese adults, Journal of the Acoustical Society of America, 118, 3267-3278

Iverson, P., Pinet, M. and Evans, B. G. 2011, Auditory training for experienced and inexperienced second-language learners: Native French speakers learning English vowels, Applied Psycholinguistics, 33, 145-160

Lambacher, S. G., Martens, W. L., Kakehi, K., Marasinghe, C. A. and Molholt, G. 2005, The effects of identification training on the identification and production of American English vowels by native speakers of Japanese, Applied Psycholinguistics, 26, 227-247

Pruitt, J. S., Jenkins, J. J. and Strange, W. 2006, Training the perception of Hindi dental and retroflex stops by native speakers of American English and Japanese, Journal of the Acoustical Society of America, 119, 1684-96. 